\title{
Serum Sirtuin 1, 3 and 6 Levels in Acute Myocardial Infarction Patients
}

\author{
Emrullah Kızıltunç, ${ }^{10}$ Arzu Kösem, ${ }^{2}$ Can Özkan, ${ }^{1}$ Burcu Uğurlu Ilgın, ${ }^{3}$ Harun Kundi, ${ }^{1,4}{ }^{\circledR}$ Mustafa Çetin, ${ }^{1}$ Ender Ornek ${ }^{1}$ \\ TC Saglik Bakanligi Ankara Numune Egitim ve Arastirma Hastanesi - Cardiology, ${ }^{1}$ Ankara - Turkey \\ TC Saglik Bakanligi Ankara Numune Egitim ve Arastirma Hastanesi - Medical Biochemistry, ${ }^{2}$ Ankara - Turkey \\ TC Saglık Bakanlıgı Gazi Mustafa Kemal Devlet Hastanesi - Cardiology, ${ }^{3}$ Ankara - Turkey \\ Beth Israel Deaconess Medical Center - Cardiology, ${ }^{4}$ Boston, Massachusetts - USA
}

\begin{abstract}
Background: Sirtuins may act in many cellular processes like apoptosis, DNA repair and lipid/glucose metabolism. Experimental studies suggested some sirtuin types may have protective effects against endothelial dysfunction, atherosclerosis, cardiac hypertrophy and reperfusion injury. Data about sirtuins in acute myocardial infarction (AMI) patients are scarce.
\end{abstract}

Objectives: To investigate temporal changes of serum sirtuin 1,3 and 6 levels in AMI patients; to compare the serum sirtuin 1,3 and 6 levels between AMI patients and control subjects; and to investigate the association of serum sirtuin 1,3 and 6 levels with prognostic markers of AMI.

Methods: Forty patients with AMI and 40 patients with normal coronary arteries were included. Left ventricular ejection fraction (LVEF), serum proBNP, CRP, sirtuin1, sirtuin 3 and sirtuin 6 levels were processed. Peak troponin T levels, GRACE score, first day / second day sirtuin levels were recorded of AMI patients. A p value $<0.05$ was considered statistically significant.

Results: Serum sirtuin 1,3 and 6 levels in AMI patients were similar to those in normal coronary patients. No temporal change in serum sirtuin 1,3 and 6 levels were found in AMI course. No correlation was evident between the sirtuin levels and the following parameters: proBNP, CRP, peak troponin and LVEF. Baseline sirtuin 1 and 6 levels were positively correlated with reperfusion duration. Baseline sirtuin 3 levels were negatively correlated with GRACE score.

Conclusion: Serum sirtuin 1,3 and 6 levels in AMI patients were similar to those in normal coronary patients. This study does not represent evidence of the possible protective effects of sirtuin1, 3 and 6 in AMI patients. (Arq Bras Cardiol. 2019; 113(1):33-39)

Keywords: Sirtuins/drug effects; Atherosclerosis; Lipid Metabolism; Endothelium/dysfunction; Cardiomegaly; Cellular Senescence; Carcinogenesis

\section{Introduction}

Sirtuins are NAD (nicotinamide adenine dinucleotide) dependent enzymes which consist of seven members called Sirt 1-7. ${ }^{1}$ The best known function of sirtuins is deacetylation, but they can also function as mono ADP ribosyltransferase, lipoamidase, demalonylase and desuccinylase. ${ }^{2,3}$ Sirtuins are involved in several biological processes like apoptosis, cellular survival, DNA repair/cellular aging and lipid/glucose metabolism. ${ }^{4}$ The information about the functions of sirtuins in carcinogenesis, aging and inflammation is also increasing. ${ }^{4,5}$ Additionally, there exist evidences that circulating sirtuin levels may associate with frailty, reduction of body fat mass or diabetes mellitus. ${ }^{6-8}$ Contemporary knowledge about the functions of sirtuins in the cardiovascular system in health

Mailing Address: Emrullah Kızıltunç •

TC Saglik Bakanligi Ankara Numune Egitim ve Arastirma Hastanesi - Cardiology Talatpaşa Bulvarı, № 44, Altındağ, Ankara 06100 - Turkey

E-mail: e.kiziltunc@gmail.com

Manuscript received August 21, 2018, revised manuscript October 25, 2018 , accepted November 01, 2018

DOI: $10.5935 / a b c .20190114$ and disease states is also evolving. Recent experimental studies have demonstrated the possible role of sirtuins in various cardiovascular pathologies like cardiac hypertrophy, heart failure, endothelial dysfunction and atherosclerosis. ${ }^{9,10}$

Survival of the acute myocardial infarction (AMI) patients have significantly improved with the advanced catheter-based therapies and increased availability of coronary care units, but this resulted increase in heart failure population. ${ }^{11}$ Infarct size reduction is crucial to decrease the probability of clinical heart failure and to improve prognosis in AMI patients. Early reperfusion and reduction of reperfusion injury are basic management approaches to reduce the infarct size. However, there exist many variables ascertaining infarct size and only a little is known about the underlying sophisticated molecular mechanisms. Inflammation, thrombogenicity, and oxidative stress effect infarct size and prognosis. ${ }^{12-14}$ Experimental studies revealed that sirtuin 1, 3 and 6 have beneficial effects against atherosclerosis, dyslipidemia, oxidative stress, endothelial dysfunction and inflammation. In addition, sirtuin 1 and 3 may activate cardioprotective pathways in the setting of AMI. ${ }^{9}$ Therefore, serum sirtuin levels may associate with reduced myocardial infarct size and good prognostic markers in AMI patients. To the best of our knowledge, there is no data about the association between serum sirtuin 1,3 and 6 levels and prognostic markers of AMI patients in 
English-language literature. The goal of this pilot study is to investigate temporal changes of serum sirtuin 1,3 and 6 levels in AMI patients, to determine if there is any difference in serum sirtuin 1,3 and 6 levels between AMI patients and control subjects and to investigate the association between serum sirtuin 1,3 and 6 levels and prognostic markers of AMI patients like peak serum troponin levels, serum pro-BNP levels, GRACE score and post-MI LVEF.

\section{Methods}

The study protocol was approved by the local ethical committee and informed consent forms were obtained from all participants. We enrolled patients with acute ST-segment elevational MI (STEMI) and patients with normal coronary arteries in this cross-sectional study between June 2017 and November 2017. Patients with STEMI were diagnosed according to the third universal definition of myocardial infarction document. ${ }^{15}$ A primary percutaneous coronary intervention was performed and all of the STEMI patients received guideline-mediated medical therapy. GRACE risk score $^{16}$ and TIMI risk score ${ }^{17}$ of the AMI patients were calculated. The duration between symptom onset to reperfusion, location of the infarction in electrocardiogram and presence of pre-infarction angina pectoris were recorded. Serum lipid levels, renal and hepatic function test results, complete blood count results, fasting blood glucose levels, and peak troponin $\mathrm{T}$ levels were also recorded. Serum $C$ reactive protein levels and pro BNP levels were processed on the first day of ischemic insult in AMI patients. Venous samples were obtained at the admittance, 24th hour and 48th hour of the infarction for sirtuin 1,3 and 6 analysis to see whether there was any temporal change in serum sirtuin levels after myocardial infarction. Serum lipid levels, renal and hepatic function test results, complete blood count results and fasting blood glucose levels were obtained from local laboratory records and venous samples were collected for $\mathrm{C}$ reactive protein, pro BNP and sirtuin level analysis after the coronary angiography in normal coronary artery patients. Transthoracic echocardiography was performed and the LVEF, end diastolic diameter, septal and posterior wall thickness were recorded in all patients. All of the samples for sirtuin level analysis were centrifuged at $4000 \mathrm{rpm}$ for 10 minutes, serums were separated and were frozen at -80 degrees Celsius. All sirtuin 1,3 and 6 levels were processed simultaneously with human sirtuin ELISA kits (YL Biotech, Shanghai, PRC).

Excluded from the study were as follows: The patients with a past history of $\mathrm{MI}$, stable coronary artery disease, peripheral arterial disease, heart failure and any valvular heart disease, the patients with malignancy, active infection, any chronic inflammatory disease, and any chronic renal or hepatic disease.

\section{Statistical analysis}

SPSS 18.0 software for Windows (SPSS Inc. Chicago, IL) was used for analysis of data. For continuous variables, the normality of distribution was tested using Kolmogorov-Smirnov test. The results were presented as the mean \pm standard deviation for variables with normal distribution and as median (interquartile range 25-75) for variables with abnormal distribution. The statistical comparisons of continuous variables were performed using independent samples t-test or Mann-Whitney $U$ test regarding the distribution pattern. The comparisons of categorical variables were performed using Chi-square test or Fisher's exact test. While investigating the association between sirtuins and prognostic markers of AMI, the correlation coefficients and their significance were calculated using the Spearman test. In AMI patients, serial serum sirtuin 1,3 and 6 levels were processed at the admittance, first day and the second day of the infarction and the Friedman test was conducted to test whether there was a significant temporal change in serum sirtuin levels in AMI patients. A p value $<0.05$ was considered statistically significant.

\section{Results}

Forty consecutive STEMI patients and 40 consecutive control patients with normal coronary arteries were included into the study. Baseline clinical and laboratory characteristics of the patients were shown in table 1. White blood cell count, neutrophil count, serum creatinine, CRP, proBNP values and left ventricle mass index were significantly higher in the AMI group than in the control group. The control group was composed of more female patients and less smoking patients. The mean platelet count and the mean left ventricular ejection fraction were significantly higher in the control group than in the AMI group. There was no significant temporal change in serum sirtuin levels in AMI patients (Figure 1).

Clinical and laboratory features of AMI patients were shown in table 2. Fifteen patients experienced pre-infarction angina pectoris before the ischemic insult. Median peak troponin level was $41.6 \mathrm{ng} / \mathrm{L}$ in patients without pre-infarction angina and $28.2 \mathrm{ng} / \mathrm{L}$ in patients with pre-infarction angina $(p=0.202)$. Baseline serum sirtuin 1, 3 and 6 levels were similar in the patients with and without pre-infarction angina. No correlation was evident between baseline serum sirtuin levels and following parameters: CRP levels, peak troponin T levels, proBNP levels and LVEF. Baseline sirtuin 1 and 6 levels were positively correlated with reperfusion duration. On the other hand, baseline sirtuin 3 levels were significantly negatively correlated with GRACE score (Table 3).

\section{Discussion}

In this pilot study, we investigated serum sirtuin 1, 3 and 6 levels in AMI patients and patients with normal coronary arteries. We found that median serum sirtuin levels were similar in AMI patients and control patients. We observed that serum sirtuin levels did not show a significant temporal change in AMI course. There was no correlation between serum sirtuin levels and prognostic markers of AMI patients like peak troponin, proBNP, CRP and ejection fraction. Serum sirtuin 1 and 6 levels were positively correlated with reperfusion duration. In addition, we found a significant negative correlation between serum sirtuin 3 levels and GRACE score.

Atherosclerotic cardiovascular disease is the leading cause of death all over the world..$^{18}$ Primary and secondary prevention attempts are evolving to decrease the global burden of this devastating disorder. ${ }^{19}$ Atherosclerosis pathogenesis is complicated and researches on the molecular basis of 


\section{Original Article}

Table 1 - Clinical and laboratory characteristics of the study patients

\begin{tabular}{|c|c|c|c|}
\hline & Control patients $n=40$ & AMI patients $n=40$ & $\mathrm{p}$ Value \\
\hline Age, y & $59 \pm 9$ & $57 \pm 14$ & 0.481 \\
\hline Sex, Male, $n(\%)$ & $23(57.5)$ & $37(92.5)$ & $<0.001$ \\
\hline Hypertension, n(\%) & $20(50)$ & $14(35)$ & 0.175 \\
\hline Smoking, n(\%) & $8(20)$ & $27(67.5)$ & $<0.001$ \\
\hline Diabetes Mellitus, n(\%) & $16(40)$ & $11(27.5)$ & 0.237 \\
\hline Family History for CAD, n(\%) & $4(10)$ & $10(25)$ & 0.077 \\
\hline Hyperlipidemia, n(\%) & $3(7.5)$ & $5(12.5)$ & 0.456 \\
\hline Fasting blood glucose, $\mathrm{mmol} / \mathrm{L}$ & $5.8(5.3-6.7)$ & $5.9(5.4-8.2)$ & 0.164 \\
\hline Creatinine, $\mu \mathrm{mol} / \mathrm{L}$ & $75 \pm 16$ & $84 \pm 14$ & 0.006 \\
\hline Total cholesterol, mmol/L & $4.9 \pm 1.0$ & $4.8 \pm 0.9$ & 0.675 \\
\hline $\mathrm{HDL}, \mathrm{mmol} / \mathrm{L}$ & $1.1 \pm 0.3$ & $1.0 \pm 0.2$ & 0.101 \\
\hline LDL, mmol/L & $2.7 \pm 0.9$ & $2.9 \pm 0.8$ & 0.392 \\
\hline Triglyceride, $\mathrm{mmol} / \mathrm{L}$ & $4.3 \pm 2.2$ & $3.9 \pm 2.2$ & 0.385 \\
\hline Hemoglobin, gr/dl & $14.6 \pm 1.9$ & $14.7 \pm 1.8$ & 0.904 \\
\hline Platelet count, ${ }^{*} 10^{3}$ & $279 \pm 70$ & $229 \pm 52$ & 0.001 \\
\hline WBC, ${ }^{*} 10^{3}$ & $8.5 \pm 1.9$ & $11.6 \pm 3.1$ & $<0.001$ \\
\hline Neutrophil count, ${ }^{*} 10^{3}$ & $5.1 \pm 1.4$ & $8.8 \pm 3.1$ & $<0.001$ \\
\hline Lymphocyte count, ${ }^{*} 10^{3}$ & $2.6 \pm 0.9$ & $2.0 \pm 0.9$ & 0.001 \\
\hline Monocyte count, ${ }^{*} 10^{3}$ & $0.8 \pm 0.9$ & $0.7 \pm 0.3$ & 0.581 \\
\hline CRP, nmol/L & $28.5(9.5-57.1)$ & $85.7(38.1-180.9)$ & $<0.001$ \\
\hline proBNP,ng/L & $48.8(24.2-113.0)$ & $500.9(282.0-1309.0)$ & $<0.001$ \\
\hline \multicolumn{4}{|l|}{ Sirtuins,ng/ml } \\
\hline Sirtuin 1 basal & $2.74(2.30-3.64)$ & $2.53(2.06-3.21)$ & 0.192 \\
\hline Sirtuin 1 first day & NA & $2.24(1.89-2.89)$ & \\
\hline Sirtuin 1 second day & NA & $2.08(1.55-3.18)$ & \\
\hline Sirtuin 3 basal & $2.62(2.16-3.34)$ & $2.40(1.29-3.29)$ & 0.204 \\
\hline Sirtuin 3 first day & NA & $2.46(1.37-2.97)$ & \\
\hline Sirtuin 3 second day & NA & $2.30(1.36-3.55)$ & \\
\hline Sirtuin 6 basal & $1.13(0.89-2.25)$ & $1.00(0.78-1.37)$ & 0.172 \\
\hline Sirtuin 6 first day & NA & $1.16(0.87-1.56)$ & \\
\hline Sirtuin 6 second day & NA & $1.19(0.85-1.80)$ & \\
\hline $\mathrm{EF}, \%$ & $64 \pm 2$ & $47 \pm 8$ & $<0.001$ \\
\hline LVMI, $\mathrm{gr} / \mathrm{m}^{2}$ & $87.3 \pm 14.6$ & $94.4 \pm 16.3$ & 0.044 \\
\hline
\end{tabular}

atherosclerosis proceed all over the world. In this context, this study evaluated the implication of serum sirtuin 1, 3 and 6 levels in AMI patients. Preliminary data about the sirtuins in cardiovascular diseases were obtained from experimental studies. It was demonstrated that sirtuin 1 exhibits antiatherogenic properties via acting on endothelium by endothelial nitric oxide synthase activation and reducing macrophage foam cell formation by nuclear factor $k B$ inhibition. $^{20,21}$ Another study showed that sirtuin 1 decrease serum LDL cholesterol levels. ${ }^{22}$ It was found that sirtuin 1 have antithrombotic effect in addition to the other atheroprotective effects that were mentioned above. ${ }^{23}$ Oxidative stress plays an important role in the pathogenesis of atherosclerosis and previous studies reported the antioxidant role of sirtuin $3.24,25$ Sirtuin 6 has anti-inflammatory and LDL lowering features. ${ }^{26,27}$ Human data about sirtuins and cardiovascular disorders are limited. Gorenne et al. ${ }^{28}$ reported that sirtuin 1 expression is reduced in human carotid atherosclerotic plaque. ${ }^{28}$ 


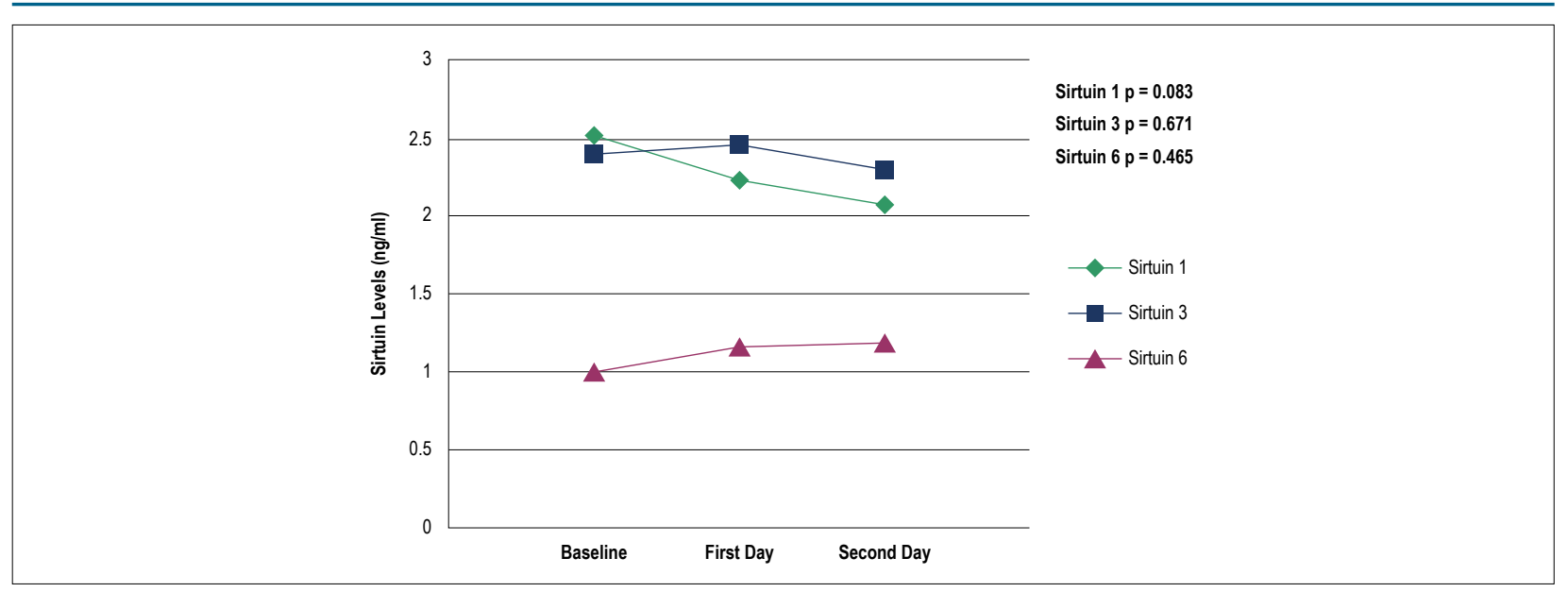

Figure 1 - Baseline, first day and second day median serum sirtuin levels of the acute myocardial infarction patients. Temporal changes of serum sirtuin 1,3 and 6 were statistically insignificant in acute myocardial infarction course.

Table 2 - Clinical and laboratory features of AMI patients $(n=40)$

\begin{tabular}{lc}
\hline Presence of Pre-infarction Angina, $\mathrm{n}(\%)$ & $15(37.5)$ \\
\hline Time to Perfusion (minutes) & $225(120-300)$ \\
GRACE Score & \\
In hospital mortality & $126(104-149)$ \\
6. month mortality & $101(77-124)$ \\
In hospital Ml/mortality & $188(151-209)$ \\
6. month Ml/mortality & $148(121-167)$ \\
Killip Class & \\
1 & \\
2 & $38(95)$ \\
3 & $1(2.5)$ \\
TIMI Risk Score & $1(2.5)$ \\
Troponin T Levels(ng/L) & $2(1-4)$ \\
Baseline & \\
First day & \\
Second day & $4.67(1.00-34.27)$ \\
Peak & $28.50(7.08-58.70)$ \\
MI Location n(\%) & $14.11(6.46-39.95)$ \\
Anterior & $30.18(10.53-63.40)$ \\
Inferior & \\
Lateral & \\
Infarct Related Artery n(\%) & $17(42.5)$ \\
LAD & $22(55)$ \\
CX & $1(2.5)$ \\
RCA & \\
AMI: acute myocardial infarction; CX: left circumflex artery; LAD: left \\
anterior descending artery; Ml: myocardial infarction; $R C A:$ right coronary \\
\end{tabular}

Breitenstein et al. ${ }^{29}$ found that peripheral monocyte sirtuin 1 expression was lower in coronary artery disease patients compared to healthy subjects. ${ }^{29}$ Judging by our results, this study may arise a debate about the protective functions of circulating sirtuin 1, 3 and 6 in cardiovascular disorders. The first issue is about the association between atherosclerosis and sirtuins. In our study population, median serum sirtuin 1, 3 and 6 levels did not significantly differ between the groups. According to our knowledge, this study is the first one comparing 'serum' sirtuin levels between normal coronary artery patients and AMI patients. There exist several studies evaluating sirtuin 1 levels in human atherosclerosis using different methodological manners. Breitenstein et al. ${ }^{29}$ measured sirtuin 1 mRNA levels in peripheral monocytes and Gorenne et al. ${ }^{28}$ measured sirtuin 1 mRNA and protein expression amount in human carotid endarterectomy materials. ${ }^{28}$ While both of these studies found a negative association between atherosclerosis and sirtuin expression, Kilic et al. ${ }^{30}$ found that serum sirtuin 1 levels were higher in stable CAD patients than in the control patients. ${ }^{30}$

By taking all these data together, the causality between sirtuins and human atherosclerosis needs more investigation. Additionally, we firstly investigated the temporal change of serum sirtuin 1, 3 and 6 levels in the AMI course and did not found any significant change between admission, first and second day of AMI. Another issue needs to be addressed is the association between inflammation and sirtuin levels. In our study, serum sirtuin 1 and 3 levels were not correlated and serum sirtuin 6 levels were positively correlated with serum CRP levels, a widely accepted inflammatory marker. As we mentioned above, experimental studies suggested that sirtuins have anti-inflammatory effects and this is hypothesized as one of the mechanisms for atheroprotection. ${ }^{31}$ We expected to find a negative association between sirtuins and CRP, but the results were not concordant with our hypothesis. Although sirtuin 6 has an anti-inflammatory feature, a possible positive regulatory role for SIRT6 in the induction of pro-inflammatory cytokine expression is evident both in innate and adaptive immune cells. Thus our positive correlation finding between sirtuin 6 and CRP can be interpreted in this context. ${ }^{32}$ 


\section{Original Article}

Table 3 - Correlation analysis of prognostic variables of AMI patients with sirtuins

\begin{tabular}{lccc}
\hline & Baseline Sirtuin 1 & Baseline Sirtuin 3 & Baseline Sirtuin 6 \\
\hline TIMI Score (Spearman's Rho/p) & $0.109 / 0.508$ & $-0.093 / 0.574$ & $0.015 / 0.930$ \\
GRACE Score (Spearman's Rho/ p) & & & \\
$\quad$ In hospital mortality & $-0.003 / 0.983$ & $-0.478 / 0.002$ & $-0.115 / 0.486$ \\
6. month mortality & $-0.001 / 0.997$ & $-0.137 / 0.406$ \\
$\quad$ In hospital MI/mortality & $0.045 / 0.785$ & $-0.351 / 0.028$ & $0.041 / 0.805$ \\
6. month MI/mortality & $0.021 / 0.901$ & $-0.509 / 0.001$ & $-0.016 / 0.922$ \\
proBNP, (Spearman's Rho/ p) & $0.294 / 0.073$ & $-0.501 / 0.001$ & $0.108 / 0.517$ \\
Peak Troponin T (Spearman's Rho/ p) & $-0.107 / 0.518$ & $-0.137 / 0.412$ & $0.012 / 0.942$ \\
Time to Perfusion (Spearman's Rho/ p) & $0.331 / 0.037$ & $-0.259 / 0.111$ & $0.312 / 0.050$ \\
CRP, (Spearman's Rho/ p) & $0.312 / 0.053$ & $-0.249 / 0.121$ & $0.357 / 0.026$ \\
EF, (Spearman's Rho/ p) & $-0.009 / 0.956$ & $-0.029 / 0.862$ & $-0.132 / 0.419$ \\
\hline
\end{tabular}

AMI: acute myocardial infarction; BNP: brain natriuretic peptide; CRP: c reactive protein; EF: ejection fraction; TIMI: thrombolysis in myocardial infarction.

Our findings regarding the association between sirtuins and the parameters reflecting infarction size like peak troponin, ejection fraction and pro BNP levels were also remarkable. Opening the occluded artery and restoring the blood flow to the myocardium is the mainstay of the AMI management. However, it was suggested that restoration of blood flow may account for further myocardial damage and it is termed as reperfusion injury. ${ }^{33}$ Microvascular obstruction, myocyte hypercontracture and contraction band necrosis, free radical generation and inflammatory cell accumulation are the proposed mechanisms for reperfusion injury. ${ }^{34}$

Ischemic preconditioning, which reduces reperfusion injury can be defined as transient, sublethal ischemic episodes rendering the myocardium more resistant to a sustained, lethal ischemic period. Since first described by Murry et al., ${ }^{35}$ ischemic preconditioning has become the most relevant entity against reperfusion injury. Ischemic preconditioning requires complex intracellular molecular interactions and the molecular mechanism is still unclear. ${ }^{36}$ It is suggested that some sirtuin types reduces ischemia reperfusion injury. In an experimental study, inhibition of sirtuin 1 resulted in reduction of bakuchiol induced cardioprotection in rat hearts. ${ }^{37}$ Opening of the mitochondrial permeability transposition pores are crucial in the pathophysiology of ischemia reperfusion injury. Sirtuin 3 deacetylates the regulatory component of the mitochondrial permeability transposition pore and sirtuin 3 deficient mice myocytes exhibited an increase in mitochondrial swelling due to increase in the number of opened mitochondrial permeability transposition pores..$^{38}$ In this study, we investigated whether there was any association between serum sirtuin levels and infarct size markers. There was no correlation between serum sirtuin levels and peak troponin levels, LVEF and pro BNP levels in AMI patients. These findings reflect that serum sirtuin 1, 3 and 6 levels were not associated with the infarct size. Pre infarction angina pectoris can be used as the surrogate marker of ischemic preconditioning in daily practice and is associated with reduced peak troponin levels in $\mathrm{AMI}$ patients. ${ }^{39}$ Although the number of subjects were low to make a firm conclusion, no difference was evident about serum sirtuin 1, 3 and 6 levels in the patients with and without pre infarction angina.
Finally, we should mention about the association between serum sirtuin 3 levels and GRACE score, and between reperfusion duration and serum sirtuin $1 / 6$ levels. GRACE score is a well-known and validated risk score for morbidity and mortality in AMI patients. ${ }^{16}$ Although we could not find any correlation between serum sirtuin 3 levels and prognostic markers like peak troponin, pro-BNP and LVEF, the negative correlation between serum sirtuin 3 levels and GRACE score may be an inspiration for further studies to investigate the role of serum sirtuin 3 levels for the risk assessment in AMI patients. We found a positive correlation between serum sirtuin 1/sirtuin 6 levels and reperfusion duration. The duration between symptom onset and reperfusion was not a marker of mortality in STEMI patients. ${ }^{40}$ Although correlated with reperfusion duration positively, sirtuin 1 and sirtuin 6 levels were not correlated with prognostic markers in AMI patients.

There are some limitations of our study. The small sample size seems the most important limitation of the study. The other important limitation is the methodological differences with previous studies. In previous studies, sirtuin analysis was performed with specimens from atherosclerotic plaques or peripheral mononuclear cells. Sirtuin mRNA levels and protein expression levels were evaluated in those studies. In our study, we directly measured serum sirtuin levels. Although our methodology was not the same with other studies, we firstly investigated serum sirtuin levels in our particular study population. This can be accepted as the originality of our study.

\section{Conclusion}

Serum sirtuin 1, sirtuin 3 and sirtuin 6 levels did not significantly differ between AMI patients and patients with normal coronary arteries. No temporal change was found in the serum levels of these sirtuins in the AMI course and there was no correlation between the serum levels of these sirtuins and the parameters reflecting myocardial infarct size like peak troponin level, LVEF and pro-BNP. We detected a negative correlation between sirtuin 3 and GRACE score, as a secondary finding. Cardioprotective role of serum sirtuin 1, 3 and 6 needs more investigation in AMI patients. 


\section{Author contributions}

Conception and design of the research: Kızıltunç $E$, Kösem A, Çetin M, Ornek E; Acquisition of data: Kızıltunç E, Kösem A, Özkan C, Ilgın BU, Kundi H; Analysis and interpretation of the data: Kızıltunç E, Kösem A, Kundi $H$, Çetin M, Ornek E; Statistical analysis: Kızıltunç E, Kundi H; Obtaining financing: Kızıltunç E, Özkan C, Ilgın BU; Writing of the manuscript and Critical revision of the manuscript for intellectual content: Kızıltunç E, Kösem A, Özkan C, Ilgın BU, Kundi $\mathrm{H}$, Çetin M, Ornek E.

\section{Potential Conflict of Interest}

No potential conflict of interest relevant to this article was reported.

\section{References}

1. Mei Z, Zhang X, Yi J, Huang J, He J, Tao Y. Sirtuins in metabolism, DNA repair and cancer. J Exp Clin Cancer Res. 2016;35(1):182.

2. Du J, Zhou Y, Su X, Yu JJ, Khan S, Jiang H, et al. Sirt5 is a NADdependent protein lysine demalonylase and desuccinylase. Science. 2011;334(6057):806-9

3. Mathias RA, Greco TM, Oberstein A, Budayeva HG, Chakrabarti R, Rowland EA, et al. Sirtuin 4 is a lipoamidase regulating pyruvate dehydrogenase complex activity. Cell. 2014;159(7):1615-25.

4. Schiedel M, Robaa D, Rumpf T, Sippl W, Jung M. The Current State of $\mathrm{NAD}(+)$-Dependent Histone Deacetylases (Sirtuins) as Novel Therapeutic Targets. Med Res Rev. 2018;38(1):147-200.

5. Carafa V, Rotili D, Forgione M, Cuomo F, Serretiello E, Hailu GS, et al. Sirtuin functions and modulation: from chemistry to the clinic. Clin Epigenetics. 2016 May;8:61.

6. Kumar R, Mohan N, Upadhyay AD, Singh AP, Sahu V, Dwivedi S, et al. Identification of serum sirtuins as novel noninvasive protein markers for frailty. Aging Cell. 2014;13(6):975-80.

7. Mariani S, Fiore D, Persichetti A, Basciani S, Lubrano C, Poggiogalle E, et al. Circulating SIRT1 Increases After Intragastric Balloon Fat Loss in Obese Patients. Obes Surg. 2016;26(6):1215-20.

8. Doulamis IP, Tzani AI, Konstantopoulos PS, Samanidis G, Georgiopoulos G, Toutouzas KP, et al. A sirtuin 1/MMP2 prognostic index for myocardial infarction in patients with advanced coronary artery disease. Int J Cardiol. 2017 Mar;230:447-53.

9. Winnik S, Auwerx J, Sinclair DA, Matter CM. Protective effects of sirtuins in cardiovascular diseases: from bench to bedside. Eur Heart J. 2015;36(48):3404-12

10. Sosnowska B, Mazidi M, Penson P, Gluba-Brzozka A, Rysz J, Banach M The sirtuin family members SIRT1, SIRT3 and SIRT6: Their role in vascular biology and atherogenesis. Atherosclerosis. 2017 Oct;265:275-82.

11. Ibanez B, James S, Agewall S, Antunes MJ, Bucciarelli-Ducci C, Bueno H, et al. 2017 ESC Guidelines for the management of acute myocardial infarction in patients presenting with ST-segment elevation: The Task Force for the management of acute myocardial infarction in patients presenting with STsegment elevation of the European Society of Cardiology (ESC). Eur Heart J. 2018;39(2):119-77.

\section{Sources of Funding}

This study was funded by Ankara Numune Training and Research Hospital.

\section{Study Association}

This study is not associated with any thesis or dissertation work.

\section{Ethics approval and consent to participate}

This study was approved by the Ethics Committee of the Ankara Numune Training and Research Hospital under the protocol number E-17-1399. All the procedures in this study were in accordance with the 1975 Helsinki Declaration, updated in 2013. Informed consent was obtained from all participants included in the study.

12. Patel MR, Mahaffey KW, Armstrong PW, Weaver WD, Tasissa G, Hochman JS, et al. Prognostic usefulness of white blood cell count and temperature in acute myocardial infarction (from the CARDINAL Trial). Am J Cardiol. 2005;95(5):614-8.

13. Hori M, Nishida K. Oxidative stress and left ventricular remodelling after myocardial infarction. Cardiovasc Res. 2009;81(3):457-64.

14. Brown AJ, Mccormick LM, Gajendragadkar PR, Hoole SP, West NE. Initial SYNTAX score predicts major adverse cardiac events after primary percutaneous coronary intervention. Angiology. 2014;65(5):408-12

15. Thygesen K, Alpert JS, Jaffe AS, Simoons ML, Chaitman BR, White HD et al. Third universal definition of myocardial infarction. Eur Heart J. 2012;33(20):2551-67

16. Fox KA, Dabbous OH, Goldberg RJ, Pieper KS, Eagle KA, Van de Werf F, et al. Prediction of risk of death and myocardial infarction in the six months after presentation with acute coronary syndrome: prospective multinational observational study (GRACE). BMJ. 2006;333(7578):1091.

17. Morrow DA, Antman EM, Parsons L, de Lemos JA, Cannon CP, Giugliano RP, et al. Application of the TIMI risk score for ST-elevation MI in the National Registry of Myocardial Infarction 3. JAMA. 2001;286(11):1356-9.

18. Gaziano JM. Global Burden of Cardiovascular Disease. In: Libby P,Bonow RO,Mann DL,Zipes DP (Ed). Braunwald's Heart Disease, 8 ed. Philedelphi a:Elsevier;2008.p.1-23.

19. Piepoli MF, Hoes AW, Agewall S, Albus C, Brotons C, Catapano AL, et al 2016 European Guidelines on cardiovascular disease prevention in clinical practice: The Sixth Joint Task Force of the European Society of Cardiology and Other Societies on Cardiovascular Disease Prevention in Clinica Practice (constituted by representatives of 10 societies and by invited experts)Developed with the special contribution of the European Association for Cardiovascular Prevention \& Rehabilitation (EACPR). Atherosclerosis. 2016 Sep;252:207-74.

20. Zhang QJ, Wang Z, Chen HZ, Zhou S, Zheng W, Liu G, et al. Endotheliumspecific overexpression of class III deacetylase SIRT1 decreases atherosclerosis in apolipoprotein E-deficient mice. Cardiovasc Res. 2008;80(2):191-9.

21. Stein S, Lohmann C, Schäfer N, Hofmann J, Rohrer L, Besler C, et al. SIRT1 decreases Lox-1-mediated foam cell formation in atherogenesis. Eur Heart J. 2010;31(18):2301-9. 


\section{Original Article}

22. Miranda MX, van Tits LJ, Lohmann C, Arsiwala T, Winnik S, Tailleux A, et al. The Sirt1 activator SRT3025 provides atheroprotection in Apoe-/- mice by reducing hepatic Pcsk9 secretion and enhancing Ldlr expression. Eur Heart J. $2015 ; 36(1): 51-9$

23. Stavrou EX, Fang C, Merkulova A, Alhalabi O, Grobe N, Antoniak S, et al. Reduced thrombosis in Klkb1-/- mice is mediated by increased Mas receptor, prostacyclin, Sirt1, and KLF4 and decreased tissue factor. Blood. 2015; 125(4):710-9.

24. Tao R, Coleman MC, Pennington JD, Ozden O, Park SH, Jiang H, et al. Sirt3mediated deacetylation of evolutionarily conserved lysine 122 regulates MnSOD activity in response to stress. Mol Cell. 2010;40(6):893-904.

25. Sundaresan NR, Gupta M, Kim G, Rajamohan SB, Isbatan A, Gupta MP. Sirt3 blocks the cardiac hypertrophic response by augmenting Foxo3a-dependent antioxidant defense mechanisms in mice. J Clin Invest. 2009;119(9):2758-71.

26. Lappas M. Anti-inflammatory properties of sirtuin 6 in human umbilical vein endothelial cells. Mediators Inflamm. 2012 Oct;2012:597514.

27. Tao R, Xiong X, Depinho RA, Deng CX, Dong XC. FoxO3 transcription factor and Sirt6 deacetylase regulate low density lipoprotein (LDL)-cholesterol homeostasis via control of the proprotein convertase subtilisin/kexin type 9 (Pcsk9) gene expression. J Biol Chem. 2013;288(41):29252-9.

28. Gorenne I, Kumar S, Gray K, Figg N, Yu H, Mercer J, et al. Vascular smooth muscle cell sirtuin 1 protects against DNA damage and inhibits atherosclerosis. Circulation. 2013;127(3):386-96.

29. Breitenstein A, Wyss CA, Spescha RD, Franzeck FC, Hof D, Riwanto M, et al. Peripheral blood monocyte Sirt1 expression is reduced in patients with coronary artery disease. PLoS One. 2013;8(1):e53106.

30. Kilic U, Gok O, Bacaksiz A, Izmirli M, Elibol-Can B, Uysal O. SIRT1 gene polymorphisms affect the protein expression in cardiovascular diseases. PLoS One. 2014;9(2):e90428.
31. Vachharajani VT, Liu T, Wang X, Hoth JJ, Yoza BK, Mccall CE. Sirtuins Link Inflammation and Metabolism. J Immunol Res. 2016 Dec;2016:8167273.

32. Galli M, Van Gool F, Leo O. Sirtuins and inflammation: Friends or foes?. Biochem Pharmacol. 2011;81(5):569-76.

33. Kloner RA. Does reperfusion injury exist in humans? J Am Coll Cardiol. 1993;21(2):537-45.

34. Cannon RO. Mechanisms, management and future directions for reperfusion injury after acute myocardial infarction. Nat Clin Pract Cardiovasc Med. 2005;2(2):88-94.

35. Murry CE, Jennings RB, Reimer KA. Preconditioning with ischemia: a delay of lethal cell injury in ischemic myocardium. Circulation. 1986;74(5):1124-36.

36. Heusch G. Molecular basis of cardioprotection: signal transduction in ischemic pre-, post-, and remote conditioning. Circ Res. 2015;116(4):674-99.

37. Feng J, Yang Y, Zhou Y, Wang B, Xiong H, Fan C, et al. Bakuchiol attenuates myocardial ischemia reperfusion injury by maintaining mitochondrial function: the role of silent information regulator 1. Apoptosis. 2016;21(5):532-45.

38. Hafner AV, Dai J, Gomes AP, Xiao CY, Palmeira CM, Rosenzweig A, et al. Regulation of the mPTP by SIRT3-mediated deacetylation of CypD at lysine 166 suppresses age-related cardiac hypertrophy. Aging (Albany NY). 2010;2(12):914-23.

39. Kiziltunc E, Abaci A, Ozkan S, Alsancak Y, Unlu S, Elbeg S, et al. The Relationship between Pre-Infarction Angina and Serum Sphingosine-1Phosphate Levels. Acta Cardiol Sin. 2014;30(6):546-52.

40. Cho YW, JangJS, Jin HY, Seo JS, Yang TH, Kim DK, et al. Relationship between symptom-onset-to-balloon time and long-term mortality in patients with acute myocardial infarction treated with drug-eluting stents. J Cardiol. 2011:58(2):143-50. 